\title{
Center of pressure (COP) measurement in patients with confirmed successful outcomes following shoulder surgery show significant sensorimotor deficits
}

\author{
Yannick J. Ehmann ${ }^{1} \cdot$ Daniel P. Berthold ${ }^{1} \cdot$ Sven Reuter ${ }^{1,2} \cdot$ Knut Beitzel $^{1,3} \cdot$ Robin Köhler $^{1} \cdot$ Fabian Stöcker $^{4}$. \\ Lukas N. Muench ${ }^{1}$. Jonas Pogorzelski ${ }^{1}$ - Marco-Christopher Rupp ${ }^{1}$ - Sepp Braun ${ }^{1,5,6}$. Andreas B. Imhoff ${ }^{1}$ (D) . \\ Stefan Buchmann ${ }^{1,7}$
}

Received: 1 April 2021 / Accepted: 16 September 2021 / Published online: 6 November 2021

(c) The Author(s) 2021

\begin{abstract}
Purpose To determine the sensorimotor and clinical function of patients with confirmed successful outcome after either undergoing acromioclavicular joint (ACJ) stabilization, Bankart repair (BR), or rotator cuff repair (RC), and to compare these measures to the contralateral, healthy side without history of previous injuries or surgeries of the upper extremity. It was hypothesized that patients of each interventional group would have inferior sensorimotor function of the shoulder joint compared to the contralateral, healthy side, while presenting with successful clinical and functional outcomes.

Methods Three intervention groups including ten patients who had confirmed successful clinical and functional outcomes after either undergoing ACJ stabilization, BR, or RC were evaluated postoperatively at an average follow-up of $31.7 \pm 11.6$ months. Additionally, a healthy control group (CG) of ten patients was included. Clinical outcomes were assessed using the Constant-Murley (CM) and American Shoulder and Elbow Surgeons (ASES) Score. Pain was evaluated using the visual analogue scale (VAS). Sensorimotor function was assessed by determining the center of pressure (COP) of the shoulder joint in a one-handed support task in supine position on a validated pressure plate.

Results Each interventional group demonstrated excellent clinical outcome scores including the CM Score (ACJ 83.3 \pm 11.8 ; BR 89.0 \pm 10.3; RC 81.4 \pm 8.8 ), ASES Score (ACJ 95.5 \pm 7.0 ; BR 92.5 \pm 9.6 ; RC 96.5 \pm 5.2 ), and VAS (ACJ 0.5 \pm 0.9 ; BR $0.5 \pm 0.8 ; \mathrm{RC} 0.5 \pm 0.8$ ). Overall, the CG showed no significant side-to-side difference in COP, whereas the ACJ-group and the BR-group demonstrated significantly increased COP compared to the healthy side (ACJ $103 \mathrm{~cm}$ vs. $98 \mathrm{~cm}, p=0.049$; BR: $116 \mathrm{~cm}$ vs. $102 \mathrm{~cm}, p=0.006)$. The RC-group revealed no significant side-to-side difference $(120 \mathrm{~cm} \mathrm{vs.} 108 \mathrm{~cm}, \mathrm{n} . \mathrm{s}$.). Conclusion Centre of pressure measurement detected sensorimotor functional deficits following surgical treatment of the shoulder joint in patients with confirmed successful clinical and functional outcomes. This may indicate that specific postoperative training and rehabilitation protocols should be established for patients who underwent surgery of the upper extremity. These results underline that sensorimotor training should be an important component of postoperative rehabilitation and physiotherapeutic activities to improve postoperative function and joint control.
\end{abstract}

Level of evidence IV.

Keywords Centre of pressure $\cdot$ COP $\cdot$ Shoulder injury $\cdot$ Rehabilitation of shoulder $\cdot$ Sensorimotor function $\cdot$ Rehabilitation

Andreas B. Imhoff

imhoff@tum.de

1 Department of Orthopedic Sports Medicine, Technical University of Munich, Ismaninger Str. 22, 81675 Munich, Germany

2 SRH University for Applied Health Sciences, Stuttgart, Germany

3 Atos Orthoparc Klinik, Cologne, Germany
4 Department of Sport and Health Sciences, TU Munich, Munich, Germany

5 Gelenkpunkt, Sport and Joint Surgery Innsbruck, Innsbruck, Austria

6 OSMI - Research Unit for Orthopaedic Sports Medicine and Injury Prevention, UMIT - Private University for Health Sciences, Medical Informatics and Technology, Hall, Austria

7 OFZ Weilheim, Weilheim, Germany 


\section{Introduction}

Postoperative limited sensorimotor function compromises sufficient joint stability along with an increased risk of future re-injuries [1-3]. The sensorimotor system has been reported to play an integrative role by mediating static and dynamic stabilizers, ensuring optimal function and stability of the shoulder joint [1, 4-7].

Recently, Edouard et al. implemented a novel testing protocol to assess the sensorimotor function of the shoulder [2]. Deficits of the afferent, central and efferent sensorimotor system, strength, or coactivation have been highlighted in patients with glenohumeral joint instability by some variations of COP [1, 2, 8, 9]. Unfortunately, detailed knowledge regarding sensorimotor shoulder function following surgery and their impact on clinical outcomes remains limited. However, this is of clinical relevance, as improved sensorimotor function may lead to better clinical outcomes, higher patient satisfaction, and reduced risk of re-injury in the long term.

The purpose of the study was to determine the sensorimotor and clinical function of patients with confirmed successful outcome, and to compare these measures to patients without history of previous injuries or surgeries of the upper extremity. It was hypothesized that patients of each interventional group would have inferior sensorimotor function of the shoulder joint compared to the contralateral side, while presenting with successful clinical and functional outcomes.

\section{Materials and methods}

Ethical approval was obtained via Human Research Determination Form to the institutional review board (IRB) of the Technical University of Munich (IRB \#64/14). A retrospective chart review was performed on patients undergoing surgery of the upper limb between 01/2012 and 06/2015 at the author's institution. Patients were eligible for inclusion if they underwent either acute (primary) ACJ reconstruction after sustaining a Rockwood IV or V injury $[10,11]$, primary rotator cuff repair, or primary Bankart repair after suffering from anterior instability of the glenohumeral joint without a significant bone loss (defined as $<15 \%$ ) [12], and had confirmed successful postoperative outcomes, defined as the absence of restrictions in clinical outcome scores and pain. Patients were excluded if they had injuries of the contralateral shoulder, neurovascular injuries, fractures, concomitant injuries to the upper limb other than ACJ instabilities (Rockwood IV, V), reconstructable isolated supraspinatus rotator cuff tears, or Bankart lesions or if they had any kind of restricting pain in their shoulders, wrists, elbows, or upper back. The first ten consecutive patients of each group to fulfill these criteria were included. Additionally, ten healthy patients without prior injuries or surgeries of the upper limb were included to serve as a control group. Minimum follow-up was 12 months.

Based on the type of injury, patients were allocated to groups: (1) arthroscopic ACJ reconstruction using a Tightrope and Endobutton (Arthrex Inc., Naples, FL, USA) repair after isolated acute ACJ injuries Rockwood type IV-V (ACJ) [13], (2) rotator cuff repair using a double-row Speedbridge configuration (Arthrex Inc., Naples, FL, USA) (RC) [14], (3) arthroscopic Bankart repair after suffering from anterior instability (BR) [15], and (4) healthy control group (CG).

\section{Clinical outcome scores}

The Constant-Murley (CM) Score was used to evaluate the level of pain and the ability to carry out normal daily activities. The American Shoulder and Elbow Surgeons (ASES) Score was used to measure shoulder pain and functional limitations in patients with musculoskeletal complaints. Pain was measured using a visual analogue scale (VAS). Previous studies have confirmed these scores in terms of reliability, validity, and responsiveness.

\section{Postoperative rehabilitation}

Each patient underwent a structured postoperative rehabilitation protocol for the first 3 months postoperatively, while shoulder braces were recommended for 6 weeks. Rehabilitation began on the first postoperative day with range of motion (ROM) being limited after rotator cuff repair as follows: weeks 1-3: passive flexion, abduction $90 / 0 / 0^{\circ}$; weeks 4-6: active flexion, abduction 90/0/0 . ROM after Bankart Repair was limited to: weeks 1-3: active flexion, abduction $45 / 0 / 0^{\circ}$; active internal/external rotation $80 / 0 / 0^{\circ}$; weeks 4-6: active flexion and abduction to $90 / 0 / 0^{\circ}$; active internal/ external rotation to $80 / 0 / 0^{\circ}$. Finally, ROM after ACJ-repair was limited: weeks 1-2: active flexion, abduction $30 / 0 / 0^{\circ}$; active internal/external rotation $80 / 0 / 15^{\circ}$; weeks $3-4$ : active flexion, abduction $45 / 0 / 0^{\circ}$; active internal/external rotation 80/0/30 ; weeks 4-6: flexion, active abduction 90/0/0 [16]. For all groups, no specific sensorimotor training was performed.

\section{Sensorimotor function testing}

The analysis of sensorimotor abilities was conducted using a static force platform (Bertec Corporation, Columbus, $\mathrm{OH}$, USA). This strain-gauge-based technology detects vertical and horizontal forces, and torques which enables calculating 
the sway of the center of pressure (COP) for the shoulder joint $[2,8]$, sampled at $100 \mathrm{~Hz}$.

Testing was conducted in a standardized setting described by Edouard et al. [2, 8]. The subjects were in a one-handed prone position with the lower part of the body supported on an adjustable height table up to the anterior superior iliac spines with the hands on the force platform, while the other hand was positioned on the belly. The height of the table was adjusted to allow the upper limbs to remain outstretched in $90^{\circ}$ of shoulder flexion with the hands placed on the platform. The elbows were in full extension, and the wrists were at $90^{\circ}$ extension to place the upper limbs at $90^{\circ}$ to the platform and the ground. The tests were performed in a noise-free environment, with no variation in luminosity. To avoid inter-tester variability, all evaluations were supervised by the same person.

A familiarization period supported on both hands with eyes open preceded each test. Tests were performed in four conditions, always in the same order: eyes open supported on the dominant side (EO-DS), eyes closed supported on the dominant side (EC-DS), eyes open supported on the nondominant side (EO-NS), and eyes closed supported on the non-dominant side (EC-NS). The recorded tests started $5 \mathrm{~s}$ after the subject maintained the test position and lasted for $25 \mathrm{~s}$. A $30 \mathrm{~s}$ period rest was given between each condition. The measurement accuracy was full centimeters.

\section{Statistical analysis}

All variables were evaluated for distribution of normality using a combination of histograms, quantile-quantile (Q-Q) plots, and Shapiro-Wilk tests. Descriptive statistics were summarized as means and standard deviations for quantitative variables and as counts and frequencies for categorical variables. The COP was summarized as medians, $25 \%$ quartiles, and $75 \%$ quartiles. The significance of mean differences between continuous, normally distributed data was evaluated using paired- and independent-samples t tests. The significance of mean differences between continuous, non-normally distributed data was evaluated using nonparametric $h$ tests (Kruskal and Wallis). The incidence between groups was assessed using Chi-square or Fisher's exact tests. Statistical significance for all comparisons was set at $P<0.05$. All analyses were performed with Stata statistical software (StataCorp. 2017. Stata Statistical Software: Release 15. College Station, TX: StataCorp LLC). A post hoc power analysis was conducted for comparison of the center of pressure measurement using a two-sided test. With an $\alpha$ of 0.05 , it was shown that the sample size in this study could achieve an adequate power of 0.92 . The sample size calculation and the power analysis were performed using G*power 3.1 .

\section{Results}

The first ten consecutive patients to meet the inclusion and exclusion criteria in each group were included. As such, the final study cohort comprised 40 patients with 10 patients in each respective group. The average time between surgery and final testing in the intervention groups was $31.7 \pm 11.6$ months. Additional demographic data for the groups and the statistical analysis are delineated in Table 1.

\section{Clinical outcome scores}

Overall, each interventional group showed excellent postoperative clinical outcome scores at a minimum follow-up of 12 months. Clinical outcomes are delineated in Table 2.

\section{Sensorimotor abilities}

The results of the COP testing are demonstrated in Table 3 . At final follow-up, there was a significant difference in COP length between the treated side and the healthy, contralateral side with eyes open for the ACJ-group $(p=0.049)$ and the BR-group ( $p=0.006$ ) (Fig. 1). However, there was no significant difference for the RC-group between the treated
Table 1 Demographic data of the patient cohort

\begin{tabular}{|c|c|c|c|c|c|c|c|c|c|c|}
\hline & \multicolumn{2}{|c|}{$\begin{array}{l}\text { Gender } \\
(n)\end{array}$} & \multicolumn{2}{|c|}{ Age surgery (y) } & \multicolumn{2}{|c|}{ Age test (y) } & \multicolumn{2}{|c|}{$\begin{array}{l}\text { Time between } \\
\text { surgery and test } \\
\text { (mo) }\end{array}$} & \multicolumn{2}{|c|}{ Affected side $(n)$} \\
\hline & $\mathrm{m}$ & $\mathrm{f}$ & Mean & SD & Mean & SD & Mean & SD & Dominant & $\begin{array}{l}\text { Non- } \\
\text { domi- } \\
\text { nant }\end{array}$ \\
\hline ACJ-group & 9 & 1 & 35.4 & 12.5 & 38.1 & 12.7 & 29.6 & 5.3 & 7 & 3 \\
\hline RC-group & 5 & 5 & 58.8 & 7.3 & 61.4 & 7.8 & 35.1 & 14.9 & 7 & 3 \\
\hline BR-group & 9 & 1 & 29.4 & 8.7 & 31.9 & 9.2 & 30.4 & 12.6 & 4 & 6 \\
\hline Control-group & 7 & 3 & - & - & 47.0 & 21.0 & - & - & - & - \\
\hline
\end{tabular}

$n$ number, $m$ male, $f$ female, $S D$ standard deviation, $y$ years, mo months, ACJ-group acromioclavicular joint group, $R C$-group rotator cuff repair group, $B R$ group Bankart repair group 
Table 2 Clinical outcome data of the patient cohort

\begin{tabular}{|c|c|c|c|c|c|c|}
\hline & \multicolumn{2}{|l|}{$\mathrm{CM}$} & \multicolumn{2}{|l|}{ ASES } & \multicolumn{2}{|l|}{ VAS } \\
\hline & Mean & SD & Mean & SD & Mean & SD \\
\hline ACJ-group & 83.3 & 11.8 & 95.5 & 7 & 0.5 & 0.9 \\
\hline RC-group & 81.4 & 8.8 & 92.5 & 9.6 & 0.5 & 0.8 \\
\hline BR-group & 89 & 10.3 & 96.5 & 5.2 & 0.5 & 0.8 \\
\hline
\end{tabular}

$A C J$-group acromioclavicular joint group, $R C$-group rotator cuff repair group, BR-group Bankart repair group, $C M$ Constant Murley Score, ASES American Shoulder and Elbow surgeons score, VAS visual analogue scale, $S D$ standard deviation; side and the healthy, contralateral side (n.s.) when assessed with eyes open or closed.

\section{Discussion}

The most important finding of the study was that significant sensorimotor deficits persist in patients after successful surgical treatment at a mean follow-up of 32 months. More importantly, these sensorimotor deficits were noted in entirely asymptomatic patients with confirmed successful postoperative clinical and functional results, confirming the stated hypothesis. These findings highlight the importance of adequate training and rehabilitation protocols in patients after undergoing surgery of the upper limb. This may be of clinical relevance, as persistent sensorimotor deficits may subsequently reduce glenohumeral stability, thus potentially increasing the risk of re-injuries.

The clinical relevance of deficits in sensorimotor function despite good outcomes in clinical scores has already been indicated in the literature [17]. Beitzel et al. recently demonstrated that elite youth javelin throwers demonstrated structural changes, even though they did not present with a history of pain or injury of the shoulder, underlining the importance of continuous postoperative rehabilitation [18]. Clinically, this may be one of the major obstacles to return to full shoulder function after injury and surgical intervention.

It can be assumed that patient-reported outcome measures ("PROs") alone may not be good enough to effectively measure the outcome of surgery of the upper extremity, especially in high-level athletes [19].

Furthermore, the data of this study demonstrated that the control group had overall low COP-results and therefore low stabilometric deviation, reproducing the results of Edouard et al. [2, 8]. Interestingly, the RC-group showed high deviations in COP measurement. This may be explained by the importance of the rotator cuff to contribute as an active stabilizer $[4,20,21]$. If this balance of forces is disturbed, glenohumeral joint kinematics change, which negatively impacts the sensorimotor function [7]. Another explanation may be the high age of the RC-group and the mostly traumatic history of ACJ injuries and glenohumeral instability that may suggest a presumably higher level of activity, which could have favorably influenced the execution of the measurement position. However, these results indicate that greater deficits in sensorimotor functionality occur after surgery, especially if the rotator cuff is involved, and may persist despite successful surgical intervention and postoperative rehabilitation.

Additionally, the injury pattern of ACJ separations does usually not cause lesions of the rotator cuff or capsule, as the most important structure for passive joint stability [20, 22, 23]. Interestingly, Witherspoon et al. demonstrated the existence of mechanoreceptors in the glenoid labrum and their importance for proprioceptive involvement in dynamic joint stabilization [24]. Clinically, Aboalata et al. showed that, despite good clinical outcomes, less than half of the patients achieved their previous level of exercise at a mean follow-up of 13 years after arthroscopic Bankart repair. This could indicate that, regardless of biomechanically sufficient restoration of the static joint stabilizers, sensorimotor deficits may persist [25].

The present study has several limitations. First, the study design does not include preoperative data regarding the sensorimotor ability of patients. However, the control group demonstrated that the contralateral side allowed for good comparability. Second, the subgroups significantly varied in their demographic composition. However, the purpose of this study was to include different types of shoulder injuries to investigate the sensorimotor impact for different structures of the shoulder joint. Third, the sample size of each subgroup is limited. However, the respective group selection was very homogeneous, which led to a very strict selection process to limit the effect of confounders. Fourth, the impact of the shoulder surgeries was compared to the contralateral, healthy side. Comparison to a healthy control group could be performed in a prospective setting to further sharpen the preliminary results. Finally, a standardized method to assess the sensorimotor function of the shoulder is yet to be determined. Other commonly used methods are the joint position sense and kinesthesia examinations [26-31].

Despite these limitations, the present data demonstrated that functional deficits even persist in entirely asymptomatic patients with confirmed successful clinical and functional 


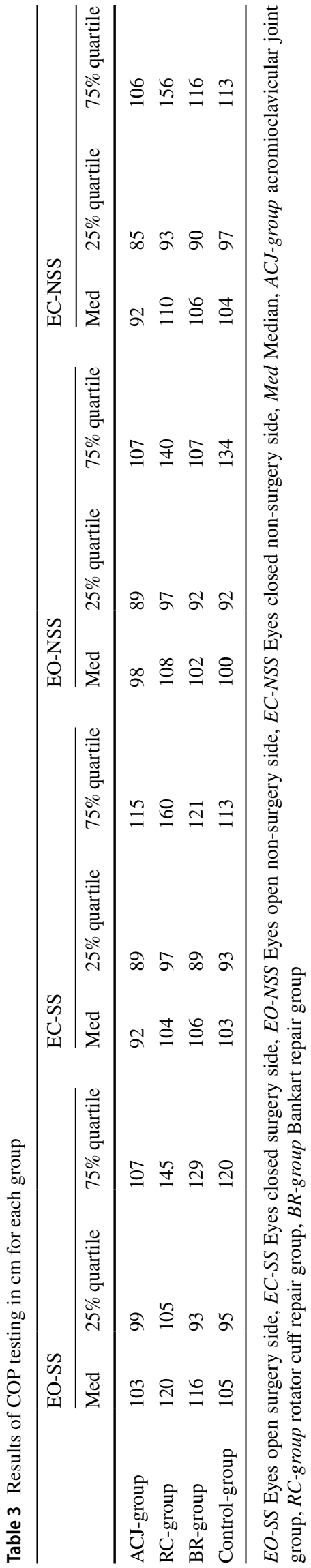

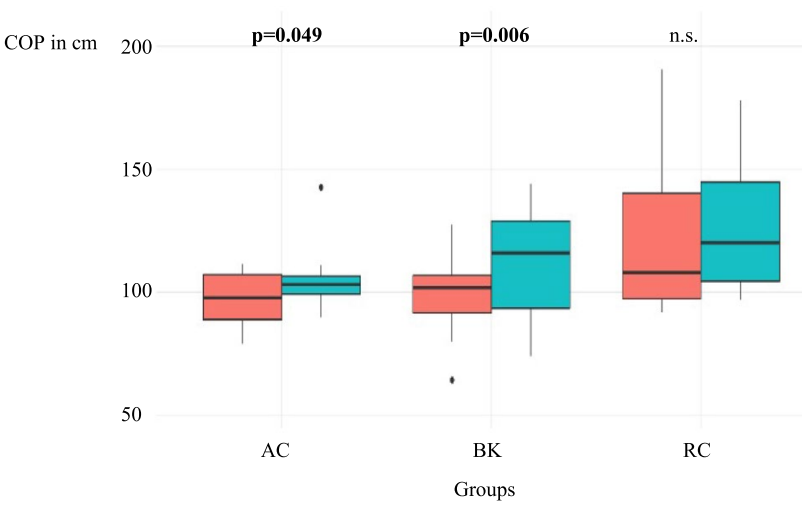

Fig. 1 Boxplot: Comparison of intervention groups with treated side (blue) vs. contralateral side (red) with eyes open in $\mathrm{cm}$

outcomes after completing postoperative rehabilitation, indicating that rehabilitation protocols may require optimization. This is of clinical importance as it may lead to better clinical outcomes, higher patient satisfaction and performance, and reduced risk of re-injury in the long term. [32] This could be implemented into day-to-day clinical practice, if rehabilitation protocols include sensorimotor training components, balancing and strengthening components, and posture optimization. [33] Consequently, this allows the surgeon to discuss the importance of postoperative sensorimotor training with the patient, as specialized instrumentation and prolonged rehabilitation protocols are needed. With more severe impairment of static and dynamic shoulder stabilizers, a greater postoperative sensorimotor deficit can be assumed.

\section{Conclusion}

Centre of pressure measurement detected sensorimotor functional deficits following surgical treatment of the shoulder joint in patients with confirmed successful clinical and functional outcomes. This may indicate that specific postoperative training and rehabilitation protocols should be established for patients who underwent surgery of the upper extremity. These results underline that sensorimotor training should be an important component of postoperative rehabilitation and physiotherapeutic activities to improve postoperative function and joint control.

Author contributions All authors contributed to the conception and design of the study. YJE, SR, KB, RK, FS were responsible for acquisition of data and writing of the manuscript. RK conducted the study as his doctoral thesis. YJE, DPB, SR, KB, RK, FS, contributed to analysis and interpretation of data. SR, KB and SB supervised RK during his thesis. LNM, JP, MR, ABI, SB, SB, contributed as important reviewers of the manuscript. All authors approved the final version of this manuscript. 
Funding Open Access funding enabled and organized by Projekt DEAL. No funding.

\section{Declarations}

Conflict of interest K.B., S. Br. and S. Bu. have received consulting fees from Arthrex. A.I. has received consulting fees from Medi and Athrosurface and royalties from Arthrex and Arthrosurface. All other authors declare that they have no conflict of interest.

IRB approval Ethical approval was obtained via Human Research Determination Form to the institutional review board (IRB) of the Technical University of Munich (IRB \#64/14).

Open Access This article is licensed under a Creative Commons Attribution 4.0 International License, which permits use, sharing, adaptation, distribution and reproduction in any medium or format, as long as you give appropriate credit to the original author(s) and the source, provide a link to the Creative Commons licence, and indicate if changes were made. The images or other third party material in this article are included in the article's Creative Commons licence, unless indicated otherwise in a credit line to the material. If material is not included in the article's Creative Commons licence and your intended use is not permitted by statutory regulation or exceeds the permitted use, you will need to obtain permission directly from the copyright holder. To view a copy of this licence, visit http://creativecommons.org/licenses/by/4.0/.

\section{References}

1. Edouard P, Degache F, Beguin L, Samozino P, Gresta G, FayolleMinon I et al (2011) Rotator cuff strength in recurrent anterior shoulder instability. J Bone Joint Surg Am 93:759-765

2. Edouard P, Gasq D, Calmels P, Ducrot S, Degache F (2012) Shoulder sensorimotor control assessment by force platform: feasibility and reliability. Clin Physiol Funct Imaging 32:409-413

3. Myers JB, Lephart SM (2002) Sensorimotor deficits contributing to glenohumeral instability. Clin Orthop Relat Res 400:98-104

4. Berthold DP, Muench LN, Bell R, Uyeki C, Zenon K, Mazzocca $\mathrm{AD}$ et al (2021) Biomechanical consequences of isolated, massive and irreparable posterosuperior rotator cuff tears on the glenohumeral joint. Obere Extrem 16:120-129

5. Dodson CC, Cordasco FA (2008) Anterior glenohumeral joint dislocations. Orthop Clin North Am 39:507-518

6. Myers JB, Lephart SM (2000) The role of the sensorimotor system in the athletic shoulder. J Athl Train 35:351-363

7. Myers JB, Oyama S (2008) Sensorimotor factors affecting outcome following shoulder injury. Clin Sports Med 27:481-490

8. Edouard P, Gasq D, Calmels P, Degache F (2014) Sensorimotor control deficiency in recurrent anterior shoulder instability assessed with a stabilometric force platform. J Shoulder Elbow Surg 23:355-360

9. Riemann BL, Lephart SM (2002) The sensorimotor system, part I: the physiologic basis of functional joint stability. J Athl Train 37:71-79

10. Beitzel K, Mazzocca AD, Bak K, Itoi E, Kibler WB, Mirzayan R et al (2014) ISAKOS upper extremity committee consensus statement on the need for diversification of the Rockwood classification for acromioclavicular joint injuries. Arthroscopy 30:271-278

11. Rosso C, Martetschläger F, Saccomanno MF, Voss A, Lacheta L, Beitzel K et al (2020) High degree of consensus achieved regarding diagnosis and treatment of acromioclavicular joint instability among ESA-ESSKA members. Knee Surg Sports Traumato Arthrosc 29:2325-2332

12. Shin S-J, Koh YW, Bui C, Jeong WK, Akeda M, Cho NS et al (2016) What is the critical value of glenoid bone loss at which soft tissue Bankart repair does not restore glenohumeral translation, restricts range of motion, and leads to abnormal humeral head position? Am J Sports Med 44:2784-2791

13. Walz L, Salzmann GM, Fabbro T, Eichhorn S, Imhoff AB (2008) The anatomic reconstruction of acromioclavicular joint dislocations using 2 TightRope devices: a biomechanical study. Am J Sports Med 36:2398-2406

14. Burkhart SS, Lo IK (2006) Arthroscopic rotator cuff repair. J Am Acad Orthop Surg 14:333-346

15. Imhoff $A B, A$, nsah $P$, Tischer $T$, Reiter $C$, Bartl $C$, Hench $M$ et al (2010) Arthroscopic repair of anterior-inferior glenohumeral instability using a portal at the 5:30-o' clock position: analysis of the effects of age, fixation method, and concomitant shoulder injury on surgical outcomes. Am J Sports Med 38:1795-1803

16. Jung C, Tepohl L, Tholen R, Beitzel K, Buchmann S, Gottfried $\mathrm{T}$ et al (2018) Rehabilitation following rotator cuff repair. Obere Extrem 13:45-61

17. Wilson KW, Popchak A, Li RT, Kane G, Lin A (2020) Return to sport testing at 6 months after arthroscopic shoulder stabilization reveals residual strength and functional deficits. J Shoulder Elbow Surg 29:107-114

18. Beitzel K, Zandt JF, Buchmann S, Beitzel KI, Schwirtz A, Imhoff $A B$ et al (2016) Structural and biomechanical changes in shoulders of junior javelin throwers: a comprehensive evaluation as a proof of concept for a preventive exercise protocol. Knee Surg Sports Traumatol Arthrosc 24:1931-1942

19. Nishigami T, Watanabe A, Maitani T, Shigetoh H, Mibu A, Wand BM et al (2021) Development and validation of a shoulder-specific body-perception questionnaire in people with persistent shoulder pain. BMC Musculoskelet Disord. https://doi. org/10.1186/s12891-12021-03944-z

20. Bigliani LU, Kelkar R, Flatow EL, Pollock RG, Mow VC (1996) Glenohumeral stability. Biomechanical properties of passive and active stabilizers. Clin Orthop Relat Res 330:13-30

21. Joseph R, Alenabi T, Lulic T, Dickerson CR (2019) Activation of supraspinatus and infraspinatus partitions and periscapular musculature during rehabilitative elastic resistance exercises. Am J Phys Med 98:407-415

22. Lawrence RL, Braman JP, Keefe DF, Ludewig PM (2020) The coupled kinematics of scapulothoracic upward rotation. Phys Ther 100:283-294

23. Willinger L, Lacheta L, Beitzel K, Buchmann S, Woertler K, Imhoff AB et al (2018) Clinical outcomes, tendon integrity, and shoulder strength after revision rotator cuff reconstruction: a minimum 2 years' follow-up. Am J Sports Med 46:2700-2706

24. Witherspoon JW, Smirnova IV, McIff TE (2014) Neuroanatomical distribution of mechanoreceptors in the human cadaveric shoulder capsule and labrum. J Anat 225:337-345

25. Aboalata M, Plath JE, Seppel G, Juretzko J, Vogt S, Imhoff AB (2017) Results of arthroscopic Bankart repair for anteriorinferior shoulder instability at 13-year follow-up. Am J Sports Med 45:782-787

26. Ager AL, Roy JS, Roos M, Belley AF, Cools A, Hébert LJ (2017) Shoulder proprioception: How is it measured and is it reliable? A systematic review. J Hand Ther 30:221-231

27. Berckmans K, Castelein B, Borms D, Palmans T, Parlevliet T, Cools A (2020) Analysis of scapular kinematics and muscle activity by use of fine-wire electrodes during shoulder exercises. Am J Sports Med 48:1213-1219

28. Berckmans KR, Castelein B, Borms D, Parlevliet T, Cools A (2021) Rehabilitation exercises for dysfunction of the scapula: 
exploration of muscle activity using fine-wire EMG. Am J Sports Med. https://doi.org/10.1177/03635465211025002

29. Herrington L, Horsley I, Rolf C (2010) Evaluation of shoulder joint position sense in both asymptomatic and rehabilitated professional rugby players and matched controls. Phys Ther Sport $11: 18-22$

30. Pötzl W, Thorwesten L, Götze C, Garmann S, Steinbeck J (2004) Proprioception of the shoulder joint after surgical repair for Instability: a long-term follow-up study. Am J Sports Med $32: 425-430$

31. Sullivan JA, Hoffman MA, Harter RA (2008) Shoulder joint position sense after thermal, open, and arthroscopic capsulorrhaphy for recurrent anterior instability. J Shoulder Elbow Surg 17:389-394

32. Yoo S, Park SK, Yoon S, Lim HS, Ryu J (2018) Comparison of proprioceptive training and muscular strength training to improve balance ability of taekwondo poomsae athletes: a randomized controlled trials. J Sports Sci Med 17:445-454

33. Leal Del Ojo P, Floría P, Harrison AJ, Gómez-Landero LA (2020) Effects of task difficulty on center of pressure excursion and its inter-trial variability in acrobatic gymnastics pyramid performance. Sports Biomech. https://doi.org/10.1080/14763141.2020. 1770322

Publisher's Note Springer Nature remains neutral with regard to jurisdictional claims in published maps and institutional affiliations. 